\title{
La construcción social de la infancia y el reconocimiento de sus competencias*
}

\author{
The social construction of childhood and the \\ recognition of their competences
}

\author{
Marco Fidel Chica** \\ Ana Lucía Rosero Prado***
}

RECIBIDO: 15 DE AGOSTO DE 2012 • ACEPTADO: 30 DE OCTUBRE DE 2012

Resumen. En el presente artículo se describe la concepción de infancia como una construcción social, a partir de la cual se denota el lugar preferencial o no que han ocupado los niños y las niñas dentro de la sociedad y las expectativas y relaciones con las personas adultas. Se presentan el aprendizaje, la educación y la formación, como espacios para la producción de significaciones, sensaciones y acciones que

* $\quad$ Artículo de investigación del proyecto titulado: «Mecanismos de influencia educativa en la enseñanza y el aprendizaje de la argumentación en niños y niñas de 5 a 6 años del nivel de transición de la Educación Preescolar». Grupo de Investigación: Educación y Pedagogía: Saberes, imaginarios e intersubjetividades - COL0076839. Línea: Educación y Pedagogía. Centro de Estudios Avanzados en Niñez y Juventud de la alianza Universidad de Manizales - Cinde.

** Doctor en Educación. Magister en Educación y Desarrollo Social, Especialista en Educación Personalizada, Especialista en Gestión Pública, Licenciando en Filosofía y Letras. Docente Investigador del Doctorado en Ciencias Sociales, Niñez y Juventud de la Alianza CINDE - Universidad de Manizales. Decano de la Facultad de Educación de la Universidad de San Buenaventura, sede Bogotá, Colombia. E-mail: mfchica@usbbog.edu.co

** Estudiante del Doctorado Niñez y Juventud de la Alianza CINDE- Universidad de Manizales. Magister en Educación Especial. Énfasis en Dificultades en el Aprendizaje, Especialista en Docencia Universitaria, Licenciada en Filosofía y letras, Licenciada en Educación Preescolar. Maestra Normal Superior Farallones de Cali, Universidad Santiago de Cali y Universidad del Valle, Colombia. E-mail: lucharop83@yahoo.com 
viabilizan el desarrollo de las competencias que hacen posible que los niños y las niñas elaboren conocimientos, prácticas comunicativas de convivencia y desde ahí, posteriormente, encuentren la razón de su existencia humana y de la transformación individual y colectiva.

Palabras Clave. Infancia; competencias; aprendizaje; educación; formación.

\begin{abstract}
This article describes the conception of childhood as a social construction from which is denotes the existence or nonexistence of a preferential place that have children in society and the expectations and relationships with adult people. Presenting of learning, education and training as spaces to produce meaning, feelings and actions that make possible development of skills that enable children to elaborate knowledge, communicative practices of coexistence and from there, find the because of his human existence and individual and collective transformation.
\end{abstract}

Keywords. Childhood, skills, apprenticeship, education, formation.

\title{
Introducción
}

Los niños y niñas, durante la etapa de la infancia, adquieren competencias a nivel social, afectivo, cognitivo, comunicativo y psicomotriz, desarrolladas mediante el aprender a aprender, el conocer, el hacer, a través de la interrelación con los demás. Es, así, como logran construir la estructura para la apropiación del mundo exterior, el reconocimiento de sí y el de los otros.

Su desarrollo y puesta en escena están sujetos tanto a las posibilidades y formas de acceder a estos procesos, como a las oportunidades de vinculación a los diversos tipos de representación simbólica y comunicativa de la cultura. Cabe anotar, que toda acción adversa a la naturaleza infantil afecta e impide el desarrollo de sus capacidades y, en consecuencia, el actuar en corresponsabilidad con su condición de educabilidad. Las reiteradas y ascendentes manifestaciones de choque que se presentan contra el ciclo vital de la primera infancia, han llevado a plantear reflexiones acerca de la recuperación del sentido de su vida y al replanteamiento de cómo el aprendizaje, la educación y la formación inciden de manera 
trascendental en el desarrollo de sus competencias y a su vez, en la constitución de niños y niñas ciudadanos y ciudadanas públicos con derechos y deberes; reflexión que hace emerger el interrogante sobre cómo el reconocimiento de la infancia y sus competencias determina el desarrollo de sus procesos y de su transformación.

En este texto se abordan tres aspectos: el primero, hace referencia a la construcción social de la infancia, el segundo aborda el aprendizaje, la educación y la formación como el camino para el desarrollo de las competencias de los niños y niñas y en el tercero se plantea la importancia del desarrollo de las competencias en la construcción de acciones para la transformación individual y social.

\section{El reconocimiento de la infancia como resultado de la construcción social}

Abordar el reconocimiento de la infancia exige la revisión de diversas concepciones que se han dado sobre los niños y las niñas en diferentes épocas, mediadas por las prácticas de crianza y las relaciones establecidas con las personas adultas. La infancia ha sido estudiada desde la psicología y la pedagogía, no obstante, dado que sus condiciones de vida se consideraban un acto privado, ésta no fue objeto de estudio y fue relegada durante mucho tiempo. Ulivieri (1986) y DeMause (1991) afirman que la falta de reconocimiento, por las personas adultas, para ver a los niños y niñas desde una perspectiva histórica, se da en algún sentido, porque sólo se les reconoce como parte del mundo adulto, cuando se produce en ellos un proceso de autonomía, antes de éste son desconocidos como sujetos con características específicas e historias de vida.

La concepción de infancia se construye a través de diferentes cambios históricos y ha sido abordada desde distintos aspectos: perspectiva socioeconómica por Ariés (1987), pautas de crianza por DeMause (1991) y lo pedagógico por Escolano (1980), entre otros. En Colombia sobresalen los trabajos sobre la infancia de Muñoz y Pachón (1998) y de Sáenz, Saldarriaga y Ospina (1997).

Philippe Aries (1987) plantea, desde la historiografía, que en la época romana la vida de los niños y las niñas se establecía a partir de dos momentos: uno cuando se salía del vientre materno y el otro cuando el padre levantaba la criatura. El nacimiento de los y las «infantes» no 
estaba provisto de expectativas, se consideraba connatural al matrimonio y la continuidad de esa vida estaba sujeta a una decisión tomada por el padre. Esta opción era arbitraria y la determinaban factores como: la duda frente a la paternidad, la normalidad congénita, la fortaleza y la vigorosidad. Cuando se les permitía continuar su curso de vida, se les guiaba hasta la mayoría de edad, de lo contrario, los niños y niñas eran abandonados, vendidos o sacrificados.

Más adelante surgen leyes que prohíben el abandono, muerte o desaparición de los y las «infantes», las cuales eran ignoradas cuando se presentaban dificultades económicas o malformaciones congénitas. La práctica del aborto se generalizó, a pesar de su ilegalidad, y en algunas ocasiones -como en el caso del madresolterismo, el cual iba en contravía de la constitución de la familia- no merecía atención, como tampoco el abandono de los hijos e hijas.

Durante los siglos II y III, tanto la familia como los hijos adquieren un lugar sagrado y se convierten en una institución atravesada por lo moral. Dicha situación permitió derrotar tanto el infanticidio como el abandono de los niños y las niñas por parte del Estado y la iglesia.

En la edad media, fue la iglesia quien se opuso al aborto y al infanticidio, Sin embargo, la idea de la malformación física, aunque no justificaba ni el abandono, ni el sacrificio, permaneció como una contravención contra la naturaleza y como un castigo divino, generalizándose así la prohibición del aborto. La crisis económica también acentúa la práctica del abandono. Ante esta situación, la iglesia aconseja dejar abandonados a niños y niñas, en la puerta de la misma o en lugares públicos, por su seguridad, antes que matarlos. Como consecuencia de esta práctica, surgen los orfanatos, lugares alternativos para recogerlos y protegerlos de su condición de abandono.

Aries (1987) explicita que en la época medieval existía poca afectividad de las personas adultas hacia los niños y las niñas y poca aceptación de su capacidad, no sólo de razonar, sino también de su condición de ser razonables. Su procreación y desaparición pasaban desapercibidas y aquellos eran mirados como unos adultos pequeños con pocas fuerzas. Sólo cuando el niño y la niña lograban cierta autonomía, al menos en la parte de su auto cuidado, comenzaban a ser considerados como miembros de la familia. 
Su interrelación con la familia estaba sujeta a la clase social a la que pertenecían; así, se puede identificar que el proceso de formación y de educación en familia era más frecuente y fuerte en las clases menos favorecidas, dado que desde esa institución los sujetos aprendían un oficio del que luego se ocuparían, mientras que en las clases altas las relaciones familiares eran más distantes, ya que los y las infantes recibían la formación y la educación de manos de las criadas o de los cuidadores. La educación institucionalizada sólo se impartía para las clases altas.

Aproximadamente en la alta edad media, se empezó a vislumbrar una posición enérgica hacia la explotación, el abandono de los infantes, gestándose así una consciencia social frente a la necesidad de su atención y una sensibilidad hacía los y las infantes. En general, tanto la edad del régimen como la edad media se identifican por ser épocas en las que los infantes padecieron todo tipo de agravios y ultrajes, ante la mirada, el consentimiento y la aprobación de las personas adultas. En estas épocas también se afianzó la idea según la cual los niños y las niñas eran hombres y mujeres en miniatura, quebradizos e inútiles.

Aries (1986) sostiene que alrededor del siglo XIV la infancia empieza a salir del anonimato y se origina su posicionamiento en la sociedad. En el siglo XVI y XVII, época del renacimiento, se da un significativo reconocimiento a las capacidades de los y las infantes; a partir de esta nueva mirada se produce el fenómeno de la escolarización y la escuela aparece como un espacio de educación formal, desplazando la educación no formal dada al interior del hogar. De esta manera, tanto la escuela como la iglesia, el Estado y la familia, se instalan como instituciones formadoras.

La actitud y la mirada de las personas adultas frente a la infancia se ubica en un nuevo horizonte a partir del momento en que la educación se institucionaliza. Los procesos de aprendizaje ya no se dan sólo desde el seno familiar; es en esta época, cuando las familias, con el ánimo de educar a sus hijos e hijas desde una postura moralizadora y bajo la influencia de los reformadores de la iglesia, ven en la educación la mejor opción.

Es en este período histórico, argumenta Aries (1986), cuando los niños y las niñas adquieren valor en sí mismos y, a diferencia de la época medieval, empieza a presentarse una distinción entre el mundo de los niños y el de los adultos. Sus trajes, por ejemplo, eran diferentes al de las personas adultas. La preocupación por el estudio del infante se da desde 
diferentes disciplinas y el sentimiento colectivo de afectividad y ternura hacia las niñas y los niños se hace mayor, cambios que se combinaban con el establecimiento de la rigidez de las personas adultas, frente a los procesos de formación implementados.

Por primera vez, en el siglo XVIII, Rousseau, en opinión de Brüggen, citado por Echeverri (2001), considera que la infancia es una etapa decisiva en la vida de los niños y niñas, en la cual se dan particularidades frente a la forma de ver el mundo y que es además, un momento crucial para su desarrollo, en el que se garantiza o no la continuidad plena para las posteriores etapas de su desarrollo. Este autor plantea como los niños y las niñas, inician el uso de los datos que llegan por sus sentidos, para construir sensaciones que acumulan y así formar las percepciones que posteriormente ordenarán para la consolidación de las ideas y el proceso de racionalización. Finalmente, expone que la educación debe adaptarse a cada una de sus etapas, a las inteligencias, los temperamentos y los caracteres de los infantes.

Posteriormente, en la edad moderna y finales del siglo XVIII, a la infancia la atraviesan fenómenos como la pedagogización y la infantilización; niños y niñas se escolarizan y se posicionan en la sociedad como sujetos dignos de protección, amor y educación, dejando de sentirlos como una obligación; así mismo, su llegada al mundo empieza a considerarse como fuente de esperanza para la mayoría de las familias.

De otro lado, DeMause (1991), aborda la historia de la infancia bajo un enfoque psicogénico, desde los modelos de crianza y las relaciones parentales.

(...) la variación de los modelos de crianza no es igual en todos los países y en todos los medios sociales; así, la relación con la infancia es susceptible aun hoy de una amplia gama de actitudes que van desde el infanticidio a la relación empática. (....) Cualquier intento de periodizar la historia de la educación infantil debe tener en cuenta que la evolución psicogenética procede con diverso ritmo en las diversas líneas familiares y que muchos padres quedan bloqueados al nivel de modelos históricos anteriores (p. 23).

DeMause refleja en su trabajo, cómo las concepciones de la infancia están ligadas a las prácticas de crianza y al desarrollo variopinto que se 
ha dado a lo largo de la historia, entre los padres y madres con sus hijos e hijas. Según el autor, se nace en un mundo que adolece de sentido, el cual se construye en relación con los estilos de crianza; esto es, que en las relaciones entre padres, madres, hijos e hijas se evidencia todo un tejido elaborado desde los valores, sentimientos y cultura, a partir del cual se construye tanto la imagen de los y las «infantes», como la imagen que ellos y ellas construyen de las personas adultas.

Se dan así relaciones, que para el autor son de varios tipos: a) La proyección, a partir de la cual los padres les trasfieren los miedos a sus hijos, haciendo con ellos lo que temen que otros les hagan, b) La reversión, según la cual los pueden ver como sustitutos de un adulto que les hace falta, c) La empatía, mediante la cual los padres se ponen en el lugar de los niños y las niñas, haciendo propias las necesidades y los deseos de sus hijos e hijas las que busca satisfacer. Divide de esta manera DeMause, en períodos, la evolución de la crianza y la relación paternofilial: el infanticidio (antigüedad-siglo IV), el abandono (siglos IV-XIII), la ambivalencia (siglos XIV-XVII), la intrusión (siglo XIII), la socialización (siglos XIX mediados del XX) y la ayuda (mediados del siglo XX).

En el infanticidio, caracterizado por la forma como los padres y madres resolvían sus ansiedades sobre los hijos, DeMause (1991) describe cómo los niños eran abandonados en lugares apartados e inhóspitos. Más adelante en el cristianismo, se reconoce a los niños y niñas como poseedores de un alma $y$, en consecuencia, para evitar transferir sus ansiedades, los padres optan por el abandono, los entregan o bien a las amas de cría, a los conventos o las casas de los nobles. Los niños y niñas que permanecen dentro de su hogar, deben moldearse a las exigencias de las personas adultas. Esta actitud se basa en una profunda ambivalencia, tanto el infanticidio como el abandono se pueden inscribir en un tipo de relación de proyección e inversión de los padres para con los hijos e hijas.

A partir del siglo XVIII empiezan a desaparecer las relaciones de proyección y de inversión; las relaciones paterno filiales se basan en la comprensión hacia la naturaleza infantil; se genera un acercamiento para intentar controlar la voluntad y la mente de niños y niñas, para tal fin, se usan métodos de castigos con los que se pretendía mejorar la relación del padre y de la madre con aquellos, dado que eran más 
sumisos y controlados. Cabe resaltar que en esa época tanto el inicio de la instrucción, como el cuidado por parte de los padres, las madres y el surgimiento de la pediatría reducen la mortalidad infantil.

En el siglo XIX y mediados del siglo XX, las familias empiezan a tomar consciencia de la importancia que tiene la socialización de los niños y niñas, espacio en el que se buscan formas de enseñarles a vivir, acordes con su medio, satisfacer sus necesidades; es decir, empieza a darse una crianza enfocada en el proceso de formación de los infantes.

A mediados del siglo XX surge una actitud más colaborativa y participativa por parte del padre y de la madre, en las pautas de crianza. Éstas se basan en el conocimiento de las necesidades de los niños y las niñas, en el reconocimiento como seres en evolución que necesitan de múltiples atenciones que les permitan su desarrollo. El castigo empieza a humanizarse y hay una seria voluntad de los padres, las madres y la familia, no sólo para entender las condiciones de cada etapa, sino también para procurar su disfrute y satisfacción. El juego se convierte en una fuente para el desarrollo biológico, psíquico y social.

DeMause (1991) plantea, desde la teoría psicogénica, que la fuerza del cambio no ha sido generada por la tecnología, ni la ideología, ni la religión, ni la economía, sino por el entramado generado desde las interacciones entre padres e hijos. Esta teoría psicogénica ofrece un paradigma nuevo para el estudio de la historia. Con arreglo a esta teoría, el supuesto tradicional de la mente como tábula rasa se invierte y es el mundo el que se considera como tábula rasa. Cada generación nace en un mundo de objetos carentes de sentido, que sólo adquieren su significado, si el niño recibe un determinado tipo de crianza:

Tan pronto como cambia para un número suficiente de niños el tipo de crianza, todos los libros y objetos del mundo quedan descartados por inútiles, para los fines de la nueva generación, y la sociedad empieza a moverse en direcciones imprevisibles. Todavía hemos de averiguar cómo se relaciona el cambio histórico con el cambio de las formas de crianza de los niños (p. 92).

A partir del siglo XX y hasta la actualidad, se dan muchos y grandes acontecimientos en el campo social, cultural y económico que inciden sobre el ser y la consciencia de niños y niñas: el conocimiento vertigi- 
noso sobre el crecimiento y el desarrollo de los niños y niñas, originado desde saberes específicos como la psicología, la medicina, la fisiología experimental, la sociología y la antropología; los aportes de la biología y las teorías evolucionistas para comprender el funcionamiento de su cuerpo, su capacidad de adaptación y de transformación; instrumentos jurídicos generados como consecuencia de la Declaración de Ginebra (1924), la Declaración de los Derechos del Hombre (1948), la Declaración Universal de los Derechos del Niño (1959) y la Convención sobre los Derechos del Niño (1989) los cuales exigen al mundo reconocer a los niños y las niñas como sujetos de protección y derecho, y que a su vez llevan a la obligatoriedad de la enunciación y la reglamentación de leyes que favorezcan el desarrollo de los infantes. La búsqueda de igualdad de la mujer, la violencia; el desplazamiento forzado; la migración a las grandes ciudades; la reivindicación de las parejas homosexuales; los avances de la biotecnología. La transformación de la familia contemporánea en la que se genera una estructura y composición con un nuevo orden organizativo, funciones y códigos y modificación de los rangos, roles; la jerarquía entre sus miembros es transferida de acuerdo con el nivel económico y social y el acceso a la información, consumo generalizado a partir del cual los niños y las niñas no dependen de la información y la enseñanza centrada en las personas adultas, lo que viabiliza su autonomía y la construcción de una reglas alejadas de las determinadas por los adultos, entre otros.

Estos acontecimientos han modificado significativamente, tanto las relaciones de las personas adultas con los y las «infantes», como la concepción de infancia, al igual que han introducido lenta y progresivamente -aunque más aceleradamente en los europeos-, la consideración sobre la idea de que la etapa infantil es trascendental en la vida de la humanidad, en tanto las vivencias que se den en este momento son de alta influencia y de difícil reversibilidad para el desarrollo del ser humano. También toma fuerza el reconocimiento de que es en esta etapa donde se desarrollan y cuando se producen de manera vertiginosa, las conexiones neuronales, las múltiples y complejas redes de neuronas donde se fundan las estructuras básicas para el crecimiento, la adquisición de conocimiento, habilidades, actitudes esenciales para la apropiación e interacción del contexto y la resolución de los problemas; en consecuencia desde este reconocimiento, se generaliza la idea de la importancia de los estímulos adecuados para potencializar sus capacidades intelectuales, físicas y sociales. 
Hoy es evidente la distancia y frontera casi inquebrantable entre el mundo infantil y el de las personas adultas, originadas en razón a que los procesos de información ya no son exclusividad de éstas y que niñas y niños tienen acceso directo a todo tipo de información, a través de los expansivos medios de comunicación, lugar desde el que encuentran diferentes formas de relación con el conocimiento. Por tanto los medios, metodologías, estrategias, temáticas y materiales utilizados por las personas adultas para la enseñanza a los niños y niñas pierden vigencia y eficacia, permitiendo que la homogeneidad de los métodos de enseñanza formal y no formal sea superada por la multiplicidad de medios de comunicación y que a muy temprana edad adquieran conocimientos, elaboren juicios y prejuicios. De esta manera, los medios de comunicación se institucionalizan como espacios de socialización, generando nuevas formas de relación y comunicación entre personas adultas e infantes, sustituyendo espacios de manera progresiva, antes considerados exclusivos para la familia y la escuela.

Desde esta aproximación, y para cerrar este breve recorrido histórico, Narodowski (1997), muestra un panorama de las tipologías que se pueden encontrar sobre la infancia. Una infancia desrealizada la cual desde temprana edad se ubica en situación laboral o vive en la calle, asumiendo su sostenimiento y relacionándose rápidamente con las personas adultas. Una infancia hiperrealizada conformada por los niños y niñas que viven su época infantil, no al lado de la familia sino de las nuevas tecnologías que les hace usuarios de mucha información, las que día tras día ganan espacios en su vida produciendo nuevos lenguajes, hipertextos, comunicación en red a los que acceden en un solo espacio y tiempo afectando significativamente sus niveles cognitivos y formas de relación con los otros. Y una infancia no nominada que se encuentra entre estas dos tensiones.

La explicitación de la construcción de la concepción de infancia, lleva a reflexionar sobre el papel que han jugado en ésta la percepción y la relación que las personas adultas establezcan con los niños y niñas. Así mismo, como los aprendizajes, la educación y la formación, desde lo formal y lo no formal, han incidido como espacios de práctica para que de ellos y ellas puedan emerger tanto sus características accidentales como esenciales. A continuación se presentan los espacios que han y permiten el desarrollo de sus competencias. 


\section{Aprendizaje, educación y formación, espacios para el desarrollo de las competencias de los niños y niñas}

A partir de la construcción social de la infancia a través de la historia, se puede identificar que en el tiempo, niños y niñas se han visto enfrentados a asumir nuevos y grandes retos, que en la actualidad, se hacen más fuertes y por tanto les implica la adquisición de conocimientos cada vez más complejos que deben poner en escena para responder a las exigencias y dinámicas sociales que determinan su existencia, la relación con los demás, las instituciones, las ideas y los valores dominantes, la diversidad de roles, funciones y grupos sociales.

Dado que nacen al interior de una red de relaciones sociales que los inscriben en una historia, en un patrimonio cultural y que a su vez los identifica, al imperativo biológico y su condición humana, los niños y niñas deben concentrarse en atender a los intereses prácticos y a los interrogantes de su existencia. Al respecto, Vigotsky (1979), plantea que el desarrollo infantil se produce bajo condiciones de cambios dinámicos en el organismo de los procesos de crecimiento, maduración y desarrollo cultural.

Así, el crecimiento en el seno de la sociedad implica por regla general una fusión con los procesos de maduración orgánica. Ambos planos de desarrollo, el natural y el cultural, coinciden y se confunden entre sí. Werstch y Vigotsky (1988) propone que las dos líneas de cambio penetran una en la otra y forman básicamente una única línea de formación sociobiológica en la personalidad. Las y los infantes logran su autoposicionamiento, autodeterminismo y la apropiación de la cultura que construyen y prolongan en diversas direcciones y que constituye finalmente su ser; ser que expresan mediante manifestaciones culturales como patrones normas, hábitos y costumbres que decretan los rasgos de su vida. Álvarez (1997) plantea que la cultura define su comportamiento y que a partir de ésta toman posesión de significados, símbolos, diversos sistemas verbales para hacer explícitas sus capacidades cognitivas, socio-afectivas y comunicativas; estas capacidades traducidas en competencias y su puesta en escena sólo son posibles a través de procesos fundamentales como el aprendizaje, la educación y la formación.

El aprendizaje, abordado desde una perspectiva constructivista, presenta diversos enfoques y teorías, clasificadas por Coll (1990) como: una teo- 
ría genética, una teoría del desarrollo intelectual, un aprendizaje verbal significativo, una teoría del procesamiento humano de la información y una teoría sociocultural del desarrollo y el aprendizaje. Cada una de estas teorías presenta variantes en su interior, frente al proceso de aprendizaje, mas coinciden en que conciben al niño y a la niña como sujetos activos y partícipes en el aprendizaje y en la construcción del conocimiento a partir del cual interpretan, comprenden su realidad y construyen su identidad.

Según el mismo autor, el proceso de aprendizaje gira en torno a que quien aprende es quien construye su conocimiento. A través del uso de sus competencias y de saberes instituidos, el sujeto elabora estructuras de pensamiento que viabilizan la reconstrucción de un conocimiento cultural socialmente reconocido y hace el enlace entre los procesos internos de construcción de conocimiento y el saber cultural determinado. De esta manera, el conocimiento se da mediante un proceso de elaboración, durante el cual, quien aprende selecciona, organiza y transforma la información, en relación con sus conocimientos iniciales y competencias.

Desde esta perspectiva, el aprendizaje se considera entonces, como un proceso de construcción de significados y sentidos, que se da en el sujeto que aprende, en interacción con el entorno y bajo la ayuda de una persona cuya relación con el conocimiento es asimétrica. Vigotsky (1979) sostiene que: «El aprendizaje humano presupone una naturaleza social específica y un proceso, mediante el cual los niños acceden a la vida intelectual de aquellos que los rodean» (p.136), planteamiento en el que se devela y resalta también, la importancia que tienen las personas adultas en el aprendizaje y su trascendencia para acceder al proceso cultural del aprendiz. Coll (1990) al respecto afirma:

\begin{abstract}
(...) Conviene subrayar el doble sentido de la ayuda pedagógica. Por una parte, es sólo una ayuda porque el verdadero artífice del proceso de aprendizaje es el alumno; es él quien va a construir los significados y la función del profesor es ayudarle en ese contenido. Pero, por otra parte, es una ayuda sin cuyo concurso es altamente improbable que se produzca la aproximación deseada de significados que construye el alumno y los significados que representan y vehiculan los contenidos escolares (p. 448).
\end{abstract}

Esta idea, además de asignarles a la niña y al niño el papel central en el aprendizaje, destaca la influencia que poseen los contextos y la partici- 
pación de las personas adultas, en el desarrollo de las competencias. Por su parte, Vigotsky (1979) denomina a este proceso la zona de desarrollo próximo definida como:

La distancia entre el nivel real de desarrollo (alcanzado por el niño), determinado por la capacidad de resolver de manera independiente un problema, y el nivel de desarrollo potencial determinado a través de resolución de un problema bajo la guía de un adulto o en colaboración de otro compañero más capaz (p.133).

Cuando la niña y el niño llegan a esa instancia, se puede decir que logran formas de razonar, de resolver problemas, de valorizar objetos, personas, situaciones y formas de conducta entre otras, es decir, desarrollan sus competencias. A esta zona no llegan a través de ejercicios mecánicos y rutinarios, aislados y atomizados, sino a través de actividades que comprometen procesos de pensamiento como: la abstracción, la síntesis, el análisis, la generalización e inscritos preferiblemente en contextos sociales.

El aprendizaje en los niños y las niñas se produce, entonces, cuando se les suministra una ayuda específica, a través de la participación en actividades intencionadas, planificadas y sistemáticas por medio de las cuales se propicia una actividad mental constructiva. Coll (1990) afirma que la construcción del conocimiento puede analizarse desde los procesos psicológicos implicados en el aprendizaje y los mecanismos de influencia educativa susceptibles de promover, guiar y orientar dicho aprendizaje.

Lo anterior sugiere que la mediación social para la internalización de los aprendizajes -operación que inicialmente representa una actividad externa, se reconstruye y empieza a suceder al interior de la persona-, debe ser proporcionada por otras personas, instrumentos y signos que tienen significado en una cultura específica y que se originan en las prácticas socialmente organizadas.

El aprendizaje también es originado gracias a la condición de educabilidad de los niños y las niñas, la cual está mediada por sucesos socioculturales, históricos y condiciones biopsíquicas. Ésta es una condición potente en el desarrollo infantil, que se fundamenta y tiene razón de ser, en su carácter inacabado y en su potencialidad de perfectibilidad. Por su parte, Zambrano (2001) afirma que a los seres humanos les es posible la 
modificación de su condición natural (acabamiento) y, además, entrar en relación de igualdad frente al que los guía, los conduce y les muestra el camino por efecto de la educabilidad. Konisberg (1974) acentúa esta posición afirmando que «la única criatura que necesita ser educada es el hombre y que, por ello, éste logra desprenderse de su condición de animalidad» (p.39). Desde esta condición, la educación se presenta en la vida de los niños y las niñas como un proceso imprescindible para la construcción y apropiación de los conocimientos sociales, físicos, culturales y en el desenvolvimiento individual y colectivo. Reboul (1971), citado por Zambrano (2001), expone que la educación «es la acción que le permite desarrollar aptitudes físicas e intelectuales, así como sentimientos sociales, estéticos y morales con el fin de cumplir en cuanto sea posible con su tarea de hombre, la que, a la vez, es el resultado de ésta acción» (p.39). Este proceso y la interacción con la cultura, facultan a los y las infantes para que se apropien de los saberes, normas, costumbres, prácticas de sobrevivencia y convivencia, básicas para su interrelación con los demás y con el contexto; para la construcción de su subjetividad y el desarrollo de sus potencialidades, aspectos fundamentales para aprender a discernir y a actuar acorde con los sistemas y organizaciones; además, a ser interlocutores y miembros válidos dentro un contexto específico.

Los niños y niñas como seres educables, inmersos en la educación, entran al proceso de humanización y caracterizan su desarrollo individual. Se apropian de la experiencia de la sociedad, de la cultura, de la ciencia; y finalmente, la condición de educabilidad posibilita su proceso de autoformación, integración, participación reflexión y su capacidad para intuir, describir lo que ven y lo que no ven, llegar al conocimiento, es decir, desarrollar sus competencias de carácter cognitivo, comunicativo y valorativo, con las cuales se posicionan en el mundo en forma significativa y propositiva.

Como complemento del proceso educativo y con el propósito de proporcionar elementos necesarios para el desarrollo de la vida de los niños y niñas, se encuentra el proceso formativo, mediante el cual se apropian de una serie de saberes y conocimientos, -adquiridos a partir de la educación- y además, en el que desarrollan su sensibilidad para asombrarse, percibir, imaginar, crear, recrear y comprender los fenómenos de la naturaleza. Gadamer (1997) plantea que mediante la formación (Bildung), el sentido común, la reinterpretación, la capacidad del juicio y el gusto, 
se adquiere la sensibilidad para trascender y comprender la realidad y los cambios espirituales.

Así, mediante los procesos de aprendizaje, educación y formación y dadas sus capacidades para conocer, interrelacionarse y comunicarse, los niños y las niñas son capaces de hacer un algo en un momento y lugar determinado y por lo tanto, trascender y posicionarse en su existencia y cultura. Esta oportunidad y capacidad recibe la denominación de competencia, la cual inicialmente surge como una respuesta a las limitaciones que se presentaban con el concepto de aptitud (muy próxima a la idea de don), que desde la tradición psicológica hacia referencia a la capacidad de carácter innato que poseen los individuos y que se expresa en la facilidad o dificultad para manejar los números, para comunicarse en forma oral o escrita y para resolver problemas abstractos. En últimas, la humanidad era clasificada en personas que tenían o no una aptitud específica.

\section{El desarrollo de las competencias en los niños y niñas una posibilidad para su transformación}

El término de competencia se asume como una idea no acabada que se refiere a las capacidades de las personas. Al respecto, Bruner (1998) sostiene que no existe una naturaleza humana estática que no se encuentre condicionada por el medio cultural que le rodea. Para este autor, las acciones humanas son acciones situadas en un escenario concreto y en ese actuar situado se desarrollan los conocimientos y habilidades necesarios para que el individuo se ajuste dialécticamente a su entorno. A partir de ellas, se logra explicitar el comportamiento, no sólo por referencia a factores intrapsíquicos (disposiciones innatas), sino también teniendo en cuenta las acciones basadas en el contexto. Esta perspectiva tiene su origen en los años 50 con los trabajos de Chomsky (1980), autor que propone tres premisas sobre las cuales se instauraría una competencia:

1. Es un tipo de acción que continuamente se transforma y perfecciona, a partir de un inicio inexperto, para afrontar las exigencias del entorno.

2. Se relaciona con un dominio particular, que exige el despliegue de acciones específicas condicionadas por las características del medio en el que éstas se dan. 
3. Es ante todo un «saber hacer», el cual conforme se perfecciona, se va volviendo menos accesible a la consciencia.

Vale la pena anotar que las competencias se construyen desde tres niveles: el primero es la apropiación de los múltiples códigos lingüísticos propios de un saber y sus objetos de estudio; el segundo la utilización de los saberes en situaciones cotidianas o hipotéticas; y el tercero, la capacidad para argumentar su utilización.

Las competencias en los niños y niñas son susceptibles de transformarse y perfeccionarse con el tiempo; poco a poco se manifiestan en el entramado de las relaciones, en el desempeño individual y colectivo, a través de prácticas en las que se integran conocimientos, destrezas y habilidades para resolver situaciones de forma propositiva y cooperativa. Son desarrolladas con el acompañamiento de los pares, las personas adultas y desde relaciones asimétricas. Estas capacidades también les permiten hacer explícitos sus conocimientos, procesos de análisis, síntesis, abstracción, razonamiento, operaciones formales, estructuras lingüísticas y se constituyen en una oportunidad para realizar procedimientos y alcanzar la autonomía que les permite vivir de forma significativa en la cultura, aprender a hacer, a saber algo en un momento y lugar determinado. Estas se categorizan en:

a) Competencia cognitiva, con la que los niños y niñas logran construir procesos mentales para comprender fenómenos físicos, humanos y naturales; diferenciar los distintos puntos de vista e interpretar las intenciones y propósitos de las acciones de los demás; plantear opciones para resolver un problema o situación, reconocer los efectos que produce cada acción; identificar los errores de interacción y asumir la autocorrección; cuestionar la validez de cualquier creencia, afirmación o fuente de información y viabilizar los acuerdos de beneficio mutuo. Con referencia a esta capacidad Piaget (1982) plantea que los niños y las niñas poseen capacidad para elaborar procesos de toma de conciencia y plantear hipótesis. Kagan (1972) argumenta que la activación de hipótesis que desarrollan los niños y las niñas pequeños para transformar esquemas es lo que les produce extrañamiento en sus esquemas más familiares. Ordoñez, O y Bustamante L (2000) sostienen que los y las «infantes», desde temprana edad, son capaces de comprender e inferir cuando construyen sistemas de clasificaciones con lo que 
establecen categorizaciones, ordenan objetos y experiencias; de igual manera, subrayan la capacidad que tienen para realizar correspondencia de objetos, representar mentalmente, hacer anticipaciones y resolver algunos problemas de índole físico o social que se les plantean.

b) La competencia socioafectiva la cual permite a los niños y niñas interiorizar el funcionamiento de organizaciones, significados y sentidos para relacionarse con los demás; esto es, que desde el conocimiento y el pensamiento pueden actuar en función de resolver problemas, emitir juicios, proponer, argumentar y predecir los comportamientos. Villalobos (2001) las define como «las capacidades intersubjetivas configuradas por la capacidad de reconocer la información que otro posee, leer las intenciones de los otros y actuar en función de ello comienzan a establecerse bien pronto en la vida» (p. 98). Está competencia está mediada por la capacidad cognitiva y por ello niños y niñas se apropian de códigos lingüísticos y simbólicos, perciben, infieren comportamientos y eventos, diferencian lo pertinente de lo no pertinente y reconocen el significado de los comportamientos, lo cual se hace visible a través de las lecturas intangibles, de expresiones verbales y no verbales.

c) La competencia comunicativa por su parte, se convierte para los niños y las niñas en el medio de explicitación más efectivo para la formación de un sujeto cuya expresión máxima es el lenguaje, al que Halliday (1982) considera como «un hecho social que surge en la vida del hombre mediante un continuo intercambio de significados con otros significados» (p.9). Hymes (1996), desde la sociolingüística, plantea que esta capacidad no sólo se da desde el conocimiento de las reglas y signos y de las estructuras lingüísticas del lenguaje, sino también en situación de uso, en las adecuaciones que se realizan de acuerdo con las particularidades de un contexto; esto es, desde el hecho de saber qué se habla, cuándo, dónde, con quién y en qué forma. Esta competencia es considerada como una predisposición genética que se desarrolla sólo mediante la acción y relación con contextos comunicativos y pluralidad de discursos y la forma de representación más universal de pertenecer a un medio sociocultural, expresar los conocimientos y sentimientos sobre acontecimientos, fenómenos de la realidad y establecer relaciones y vínculos afectivos. 
A partir de lo anterior, se puede precisar la relación existente entre aprendizaje, educación, formación y la incidencia de estos procesos en el desarrollo de las competencias y en consecuencia en la vida de los niños y niñas; aquí se originan y conjugan las múltiples lógicas y lenguajes que les permiten ser, aprender y actuar en momentos de resolución de situaciones problémicas así como establecer relaciones personales, sociales e interactuar en espacios públicos y privados. El uso de las competencias en los y las infantes es un requerimiento fundamental para la generación y el manejo de sus emociones, manifestar asertivamente sus intereses y captar elementos comunes de participación que garantizan su transformación. Estas potencialidades les permiten el establecimiento de la vida en comunidad, como un tejido de relaciones y contribuyen a la construcción de una cultura que rompe con comportamientos estandarizados que sustentan su anonimato. Los anteriores procesos y reconocimientos validan la existencia de infancias que necesitan derrotar los desaciertos de la invisibilización infantil y las consecuencias que este fenómeno social ha dejado en las diferentes maneras de sentir, pensar, actuar de los y las infantes.

Este camino abre el espacio para reconocer en cada niño y niña su capacidad para discernir y actuar, desde su dimensión singular y su contexto cultural; restablecer el acceso a lenguajes, normas y valores con los que son identificados como miembros de una comunidad, con formas de comportamiento únicas e irrepetibles y fortalecer el desarrollo de su autonomía, autoaprendizaje, discursivos éticos que constituyen la discusión intersubjetiva y contribuyen en consecuencia a la construcción social de la infancia a partir del reconocimiento de su diversidad biopsíquica y cultural, el respeto a sus derechos la participación y la transformación de sí y de los demás.

\section{Conclusiones}

La nueva imagen de la infancia implica no sólo la apertura y creencia en sus capacidades, sino también la ruptura con la instalación y el surgimiento de asuntos como la constitución de la nueva familia, que se devela en la actualidad como un grupo heterogéneo con diversidad de estructuras, relaciones y roles, y donde es posible o no que se propicie el desarrollo de las competencias o la desinfantilización de los niños y niñas. Así mismo, otro aspecto es el referido a su inmersión en el mundo de la tecnología y la informática, en el que encuentran una cantidad 
inmensurable de información en un solo espacio, lo que afecta tanto lo que piensan y sienten y en consecuencia, genera nuevas formas de aprender y de relacionarse con las personas adultas. Paralelamente a la transformación en la familia y a los avances tecnológicos, se puede anotar que los cambios económicos y políticos han originado ideas y disputas sobre la infancia. Es evidente que estos acontecimientos han forjado prácticas y discursos que exigen el levantamiento y aceptación de las diversas formas de relaciones que pueden surgir con las personas adultas y de procesos éticos que permitan, en un nuevo tiempo y orden, el fomento de los principios de equidad, el respeto y el reconocimiento por los derechos y deberes de los niños y las niñas.

El aprendizaje, la educación y la formación, se presentan en la infancia como procesos que contribuyen al desarrollo de las competencias; habilidades con las que los niñas y niñas son capaces de apropiarse de un mundo simbólico, de la cultura, de las expresiones sociales, de formas de pensar, así como de estructurar el lenguaje e interpretar y construir realidades. Su desarrollo toma su base en la infancia y se despliega a lo largo de la vida, dota a los niños y a las niñas de conocimientos, vivencias y sentimientos con los que pueden interactuar; cuantos más escenarios de visibilización posean para desarrollar las competencias, más oportunidades tendrán para la comprensión e interpretación del mundo.

Las experiencias infantiles se convierten en el insumo que nutre sus potencialidades, contribuyen al fortalecimiento de la autonomía y a los procesos de identidad. De esta manera, la conquista de las competencias cognitivas, sociales y comunicativas en los y las infantes, tiene un propósito trascendental en tanto les permite no sólo interactuar con el medio natural y cultural, sino también poner en escena las habilidades, los conocimientos, valores y el planteamiento de opciones creativas, cuando de resolver situaciones cotidianas o problémicas se trate.

El desarrollo de los niños y niñas y la potencialización de sus competencias, esto es, el desarrollo de sus potencialidades cognitivas, sociales, psicomotrices, la capacidad de aprender, de ser, de hacer y de relacionarse, se ven afectados notablemente por el tipo de experiencias dadas en los primeros años de vida, de ahí que sea preciso y urgente que el mundo de las personas adultas reconozca y conozca las vías y factores que afecten la vida de los y las infantes y por ende el futuro de las generaciones. 
La condición excepcional de niños y niñas de ser educables, les permite entonces, construir capacidades saberes, sentimientos y acciones asertivos para conformar el tejido social, abordar diversas realidades provistas de significaciones, propuestas y argumentaciones para afrontar la vida y transformarla. Los contextos para el aprendizaje, la educación y la formación, determinan la capacidad de desarrollo de los niños y las niñas en tanto posibilitan que éstos puedan manifestar lo que saben, lo que piensan, lo que pueden hacer, reestructurar y construir nuevos esquemas que potencien su individualidad, física emocional y cognitiva y con ello generar la posibilidad para su transformación y la de su entorno.

\section{Referencias}

Álvarez. A. (1997). Hacía un currículum cultural. La vigencia de Vigotsky en la educación. Madrid: Editorial Fundación Infancia y Aprendizaje.

Ariés, P. (1986). La infancia. En Revista de Educación, 281, 5-17.

(1987). El niño y la vida familiar en el Antiguo Régimen. Madrid: Taurus.

Asamblea de las Naciones Unidas. (1924). Declaración de Ginebra. (Adoptada por la V asamblea de la Sociedad de Naciones Unidas el 24 de septiembre).

(1948). Declaración Universal de los derechos Humanos (Adoptada por la Asamblea General de Naciones Unidas el 10 de diciembre).

(1959). Declaración universal de los derechos del Niño. (Adoptada por la Asamblea General de Naciones Unidas el 20 de noviembre).

(1989). Convención sobre los derechos del niño. (Adoptada por la Asamblea General de Naciones unidas el 20 de noviembre).

Bruner, J. (1998). Actos de significado; Más allá de la revolución cognitiva. Madrid: Alianza.

Brüggen, F. (2001) «Perfectibilidad y educación: la construcción de la naturaleza en el pensamiento de Jean Jacques Rousseau» En: Echeverri Sánchez, J.A (Editor) Encuentros Pedagógicos Transculturales. Desarrollo comparado de las conceptualizaciones y expe- 
riencias pedagógicas en Colombia y Alemania. Medellín: Editorial Universidad de Antioquia, 249-262.

Coll, C. (1990). Un marco de referencia psicológico para la educación escolar: la concepción constructivista del aprendizaje y la enseñanza. En: C. Coll, J. Chomsky, N. (1980). El lenguaje y el conocimiento inconsciente. México: FCE.

DeMause, LL. (1991). La evolución de la infancia. Historia de la infancia. Madrid: Alianza Universidad.

Escolano, B. (1980). Aproximación histórico-pedagógica a las concepciones de la infancia. En Studia Pedagógica. Revista de Ciencias de la Educación-Universidad de Salamanca.

Gadamer, H-G. (1997). Verdad y Método. Volumen I Salamanca: Ediciones Sígueme.

Halliday, M. (1982). El lenguaje como semiótica social. México: Fondo de Cultura Económica.

Hymes, D. (1996). La sociolingüística y la etnografía del habla. En: Antropología social y lenguaje (Obra colectiva). Buenos Aires: Paidós.

Kagan, J. (1972). Resilience and continuity in psychological development. En A.M. Clarke y A.D.B. Clarke (Eds.), Early experience: myth and evidence. New York: Free Press.

Narodowski, M. (1999). Después de clase. Desencantos y desafíos de la escuela actual. Buenos Aires: Novedades Educativas.

Muñoz, C \& Pachón, X. (1988). Historia social de la infancia. Bogotá. 1900-1989. V. 7. Bogotá: Fundación para la promoción de la investigación y la tecnología. Banco de la República.

Ordoñez, O. \& Bustamante, L. (2000). Habilidades para la comprensión y el razonamiento científico en el niño. Una revisión bibliográfica. En Puche, R. (2000). Formación de herramientas científicas en el niño pequeño. Cali: Arango.

Piaget J, (1982). Introducción a Piaget. Pensamiento. Aprendizaje. Enseñanza. México (Original Inglés, traducción. Labinowicz, ed. 1982). 1. ${ }^{\mathrm{a}} \mathrm{ed}$.

Puche, R. (2000). Formación de herramientas científicas en el niño pequeño. Cali: Arango. 
Sáenz, J, Saldarriaga, O \& Ospina, A. (1997). Mirar la infancia: pedagogía, moral y modernidad. En: Colombia. 1903-1946. V. 2. Medellín: Colciencias, Ediciones Foro Nacional por Colombia, Ediciones UniAndes. Editorial Universidad de Antioquia.

Ulivieri, S. (1986). Historiadores y sociólogos en busca de la infancia. Apuntes.

Vygotsky, L.S. (1979). Pensamiento y lenguaje. Buenos Aires: La pléyade.

Villalobos, M. (2001). El desarrollo psicológico del niño juego, representación y capacidades del niño como sujeto. En: Puche, R. (2000). Formación de herramientas científicas en el niño pequeño. Cali: Arango 98.

Werstch, J. \& Vigotsky (1988). La formación social de la mente. Barcelona: Paidós.

Zambrano, L. (2001). Pedagogía, educabilidad y formación de docentes. Cali: Editorial Nueva Biblioteca Pedagógica. 